\title{
Rates of genetic testing in patients prescribed drugs with pharmacogenomic information in FDA-approved labeling
}

\author{
John Young $\mathbb{D}^{1} \cdot$ Kaustuv Bhattacharya $\mathbb{D}^{2} \cdot$ Sujith Ramachandran $\mathbb{D}^{2} \cdot$ Aaron Lee ${ }^{1} \cdot$ John P. Bentley $\mathbb{D}^{2}$
}

Received: 3 August 2020 / Revised: 8 December 2020 / Accepted: 15 January 2021 / Published online: 15 February 2021

(C) The Author(s), under exclusive licence to Springer Nature Limited 2021

\begin{abstract}
This study examined rates of genetic testing in two cohorts of publicly insured individuals who have newly prescribed medication with FDA pharmacogenomic labeling guidance. Genetic testing was rare $(4.4 \%$ and $10.5 \%$ in Medicaid and Medicare cohorts, respectively) despite the fact that all participants selected were taking medications that contained pharmacogenomic labeling information. When testing was conducted it was typically done before the initial use of a target medication. Factors that emerged as predictors of the likelihood of undergoing genetic testing included White ethnicity (vs. Black), female gender, and age. Cost analyses indicated higher expenditures in groups receiving genetic testing vs. matched comparators with no genetic testing, as well as disparities between proactively and reactively tested groups (albeit in opposite directions across cohorts). Results are discussed in terms of the possible reasons for the low base rate of testing, mechanisms of increased cost, and barriers to dissemination and implementation of these tests.
\end{abstract}

\section{Introduction}

Genetic testing is becoming increasingly common in applied healthcare environments, with substantial increases in use since 2012 and more than 75,000 commercial tests available by 2018 [1-4]. The purpose of these tests is often to reduce adverse drug reactions (ADRs), which have long been known to be problematic across healthcare environments (particularly as a major cause of death among hospitalized patients) [5-14]. Implementing genetic testing as a means of avoiding ADRs thus has the potential to both enhance health outcomes and provide economic savings to healthcare delivery systems $[5,7,15]$. This initiative also fits well within the recent movement toward individualized and/or precision medicine wherein adaptations to

Supplementary information The online version contains supplementary material available at https://doi.org/10.1038/s41397021-00211-1.

John Young

jnyoung1@olemiss.edu

1 Department of Psychology, University of Mississippi, University, MS, USA

2 Department of Pharmacy Administration, University of Mississippi, University, MS, USA standardized interventions may be facilitated by genetic analysis [16].

Rapid advances in pharmacogenomics research have also made testing procedures more viable and turnaround times faster. For some disease states, the realization of the potential benefits of genetic testing to healthcare systems and their patients have contributed to the diffusion of these techniques, some of which have advanced to the point of influencing policy. For example, more than 280 prescription drugs across numerous categories (e.g., psychotropics; opioids; cancer-treating agents; memory enhancers; diabetes medications; anti-hypertensives; statins) now contain U.S. Food and Drug Administration (FDA) genetic labeling information, which is most frequently deployed in an attempt to augment clinical strategies by identifying potential responders vs. non-responders and/or either preventing or curtailing ADRs [17]. This typically references direct molecular research, particularly findings that facilitate the prediction of negative drug response on the basis of polygenic tests or multianalyte assays with algorithmic analyses [17].

The impact of this actuarial, evidence-based strategy for generating labeling guidance on actual practice, however, has not yet been studied in detail. There are some examinations of the overall rate of use of genetic tests that have indicated significant fiscal expenditures, increasing use over time, and strong differences in frequency across clinical 
domains [1-4]. These studies provide useful information about base rates and the general trends in genetic testing in large healthcare systems but are unfortunately less informative about how often tests are administered to patients taking medications where genetic factors have the potential to be salient in the determination of treatment outcome (i.e., those with pharmacogenomic information in the FDAapproved labeling).

This lack of specificity may be at least partly due to the obfuscating nature of current procedural terminology (CPT) codes related to genetic testing, which have made it difficult to study the use of genetic tests in large healthcare claims databases [18, 19]. Prior to 2012 the CPTs used for genetic tests were typically generic and laboratories often submitted several single-allele codes to cover polygenic or another proprietary testing [19]. Greater specificity in taxonomy has arisen in recent years, which has been particularly evident in contemporary examinations of private healthcare databases, but ambiguity about the frequency and purposes of genetic tests remains high [1].

One potential way to address this gap in research is to use health insurance administrative claims data to identify individuals taking medications known to have FDA genetic labeling guidance. In combination with the broadest possible set of CPTs connoting genetic testing, this would allow some insight into the frequency of testing in situations where the utility is most pronounced and empirically informed. Thus, the current paper endeavored to explore this topic in healthcare claims submitted to Medicare and Medicaid in the state of Mississippi. The primary goal of the study was to determine the base rate of genetic testing in patients for whom this was potentially pharmacologically appropriate. Secondary goals were the identification of demographic variables likely to facilitate reception of genetic testing when taking a medication with specific labeling guidance and cost comparisons between groups as a function of reception and timing of genetic testing.

\section{Method}

\section{Procedure}

Administrative claims databases containing all provider claims for Medicare and Medicaid in the state of Mississippi for the calendar year 2014 were used in the study. These databases were licensed from the Centers for Medicare \& Medicaid Services (CMS) under a data use agreement (DUA\# RSCH-2017-51606), and all study procedures were approved by the institutional review board and granted a waiver of informed consent. Individuals were included in the study if they were (1) continuously eligible for coverage throughout the study period; (2) did not receive hospice services at any point; and (3) began a new prescription of one (and only one) of the target medications (i.e., from the FDA list of drugs with genetic testing labeling information) [17] between July 1, 2014 and December 1, 2014, as identified using national drug codes (NDCs). This allowed for the adequate examination of prior history and ensured a minimum follow-up period of at least 30 days. A prescription for a target medication was operationalized as being new (and represented the index date) if the individual had no prescription fills for the same medication in the previous 6 months [20]. The only exclusion criterion was dual-eligibility for both Medicare and Medicaid at any point during the study period.

Once identified as study-eligible, recorded claims for each qualifying individual were examined to discern whether or not any genetic testing was conducted. This was necessarily somewhat imprecise given the issues with CPT coding noted earlier but represented the highest possible base rate of genetic testing in association with medications that had labeling guidance (see Appendix 1 for CPTs used). Individuals were further categorized into groups receiving genetic testing prior to or after their index date, which allowed some inference as to whether testing was proactive or reactive (or put differently, likely administered in order to prospectively avoid or retrospectively contend with ADRs). In addition, rates of testing within the domain of medication applied usage (i.e., therapeutic area) were also calculated according to FDA-defined categories (corresponding to medical specialty).

Within each cohort (i.e., Medicare and Medicaid), individuals identified as having a genetic test were matched with an individual comparator without a genetic test who has prescribed the same target medication and had the same comorbidity burden during the 6-month lookback period (as measured by the Elixhauser comorbidity index) [21]. These comparators were used to compare treatment costs over the duration of the study and to examine potential predictors of genetic testing. Within-group costs were calculated as annualized, all-cause, per member per month (PMPM) costs in dollars in order to standardize comparisons and provide output likely to be interpretable in the context of a large healthcare system administration. Demographic characteristics of all eligible study participants, including age, race, and gender were also captured for subsequent analyses.

All analyses were conducted separately in Medicare and Medicaid to reflect the unique nature of the beneficiaries in these public insurance programs. All analyses were conducted using SAS version 9.4 (Cary, NC, USA). Bivariate analyses were conducted using the Cochran-Mantel-Haenszel test, paired $t$ test, chi-square test, and independent samples $t$ test for categorical and continuous variables in the matched and unmatched samples as appropriate. Conditional logistic regression models that account for the matched design were 
used to predict receipt of a genetic test. Comparison of economic burden was conducted using generalized linear models using a log link and a gamma distribution after accounting for age, race, and gender.

\section{Results}

\section{Participants-Mississippi Medicaid cohort}

A total of 34,420 Mississippi Medicaid beneficiaries met inclusion criteria, of whom 1525 (4.4\%) received some form of genetic testing. Among these individuals, there was a relatively small percentage of males $(15.8 \% ; n=241)$. The final analytic cohort included 1453 individuals with genetic testing who were matched 1:1 with a comparator group based on target medication and comorbidity burden, drawn from the pool of beneficiaries who were taking a target medication but did not receive genetic testing. Complete demographic information for both groups appears in Table 1.

\section{Patterns within and between groups in Mississippi Medicaid cohort}

The majority of individuals receiving genetic testing did so prior to the first recorded date of starting medication with genetic labeling guidance $(77.3 \% ; n=1123)$. Conditional logistic regression indicated that the odds of receiving genetic testing were significantly higher for White vs. Black beneficiaries (OR $=1.27$; 95\% CI: $1.09-1.50 ; p=0.003)$, for women vs. men $(\mathrm{OR}=2.15$; $95 \% \mathrm{CI}: 1.75-2.64 ; p<$ 0.0001 ), and for older compared to younger individuals $(\mathrm{OR}=1.02$; 95\% CI: $1.02-1.03 ; p<0.0001)$.

The adjusted, all-cause cost was found to be $\$ 927$ PMPM (95\% CI: \$867-992) among individuals receiving genetic testing versus \$788 PMPM (95\% CI: \$739-840) among those not receiving genetic testing. Comparing allcause expenditures across groups yielded significant differences for those receiving genetic testing vs. not, with higher costs for the genetic testing group $(p<0.0001)$. This effect was replicated when repeating the analysis for subgroups receiving either proactive or reactive testing in comparison to their respective matched comparators. A separate comparison of individuals receiving proactive vs. reactive testing indicated significantly higher expenditures for those tested before starting a target medication $(p<$ 0.0001). More detailed information concerning PMPM costs is depicted in Table 2. In addition, a list of all target medications and their frequency of prescription in this cohort appears in Appendix 2.

Details of the rates of genetic testing by therapeutic area can be seen in Table 3. Although most categories of use
Table 1 Mississippi Medicaid cohort demographics.

Total cohort

\begin{tabular}{lclr}
\hline & Genetic testing & $\begin{array}{l}\text { No genetic } \\
\text { testing } \\
n=33,011\end{array}$ & $p$-value \\
\hline $\begin{array}{l}\text { Race, } n(\%) \\
\quad \text { Black }\end{array}$ & $767(50.3)$ & $17,540(53.1)$ & \\
$\quad$ White & $668(43.8)$ & $13,140(39.8)$ & \\
$\quad$ Other & $90(5.9)$ & $2331(7.1)$ & \\
$\begin{array}{l}\text { Gender, } n(\%) \\
\quad \text { Male }\end{array}$ & $241(15.8)$ & $11,299(34.2)$ & \\
$\quad$ Female & $1284(84.2)$ & $21,712(65.8)$ & \\
$\begin{array}{l}\text { Age, mean (SD) } \\
\text { Elixhauser score, }\end{array}$ & $36.3(14.8)$ & $25.8(17.9)$ & $<0.00044$ \\
mean (SD) & $2.23(6.3)$ & $1.34(4.1)$ & $<0.0001$ \\
& & & \\
\hline
\end{tabular}

Final cohort with matched comparators

\begin{tabular}{lccc}
\hline & Genetic testing & $\begin{array}{l}\text { No genetic } \\
\text { testing } \\
n=1453\end{array}$ & $p$-value \\
\hline $\begin{array}{l}\text { Race, } n(\%) \\
\quad \text { Black }\end{array}$ & $728(50.1)$ & $800(55.1)$ & 0.0098 \\
$\quad$ White & $642(44.2)$ & $565(38.9)$ & \\
$\quad$ Other & $83(5.7)$ & $88(6.1)$ & \\
$\begin{array}{l}\text { Gender, } n(\%) \\
\quad \text { Male }\end{array}$ & $224(15.4)$ & $397(27.3)$ & $<0.0001$ \\
$\quad$ Female & $1229(84.6)$ & $1056(72.7)$ & \\
$\begin{array}{l}\text { Age, mean (SD) } \\
\text { Elixhauser score, } \\
\text { mean (SD) }\end{array}$ & $36.1(14.6)$ & $31.8(17.0)$ & $<0.0001$ \\
\hline & $1.72(5.3)$ & $1.72(5.3)$ & - \\
\hline
\end{tabular}

were associated with a testing rate of $\sim 5 \%$, some deviations were also notable. Oncology drugs, for example, were associated with testing $36.4 \%$ of the time (although few prescriptions for these medications were notable; $n=44$ ). Some prescriptions of a more appreciable frequency were also associated with deviation from this 5\% mark with either a higher (e.g., infectious disease, $7.9 \% ; n=2622$ ) or lower (e.g., gastroenterology, 2.9\%; $n=14,272$ ) rate of testing, indicating that some differences in category-specific clinical decision-making regarding genetic testing were likely.

\section{Participants-Mississippi Medicare cohort}

The total group of Mississippi Medicare beneficiaries meeting inclusion criteria was 37,788, of whom 3960 $(10.5 \%)$ received genetic testing. The gender distribution included a higher percentage of males than the Medicaid cohort, but still many fewer men $(27.4 \% ; n=1085)$ than women $(72.6 \% ; n=2875)$. The matched analytic cohort was selected using the same strategy described above and 
Table 2 Mississippi Medicaid adjusted all-cause per member per month (PMPM) costs.

\begin{tabular}{lllllll}
\hline Cohort & \multicolumn{2}{l}{ Genetic testing } & & & No genetic testing & \multirow{2}{*}{$p$-value } \\
\cline { 2 - 3 } & PMPM cost & $95 \%$ CI & & PMPM cost & $95 \%$ CI & \\
\hline Total sample & $927(n=1453)$ & $867-992$ & & $788(n=1453)$ & $840-739$ & $<0.0001$ \\
Proactive testing & $941(n=1123)$ & $868-1020$ & & $796(n=1123)$ & $737-859$ & $<0.0001$ \\
Reactive testing & $883(n=330)$ & $786-990$ & & $757(n=330)$ & $679-843$ & $<0.0001$ \\
\hline
\end{tabular}

The difference between proactive and reactive testing subgroups was significant; $p<0.0001$.

\begin{tabular}{|c|c|c|c|c|}
\hline \multicolumn{3}{|l|}{ Medicaid } & \multicolumn{2}{|l|}{ Medicare } \\
\hline Therapeutic area & $\begin{array}{l}\text { Number of } \\
\text { individuals receiving } \\
\text { a prescription }\end{array}$ & $\begin{array}{l}\text { Percentage } \\
\text { receiving genetic } \\
\text { testing }\end{array}$ & $\begin{array}{l}\text { Number of } \\
\text { individuals receiving } \\
\text { a prescription }\end{array}$ & $\begin{array}{l}\text { Percentage } \\
\text { receiving genetic } \\
\text { testing }\end{array}$ \\
\hline Anesthesiology & 6387 & 5.2 & 9295 & 10.1 \\
\hline Cardiology & 1541 & 5.4 & 4871 & 9.9 \\
\hline Dental & 0 & N/A & 5 & 40.0 \\
\hline Dermatology & 20 & 5.0 & 18 & 16.7 \\
\hline Endocrinology & 389 & 6.4 & 1662 & 9.1 \\
\hline Gastroenterology & 14,272 & 2.9 & 7958 & 10.6 \\
\hline Gynecology & 11 & 9.1 & 0 & N/A \\
\hline Hematology & 155 & 7.1 & 593 & 13.2 \\
\hline Infectious Disease & 2622 & 7.9 & 3443 & 11.6 \\
\hline Neurology & 2194 & 3.6 & 3799 & 9.1 \\
\hline Oncology & 44 & 36.4 & 310 & 40.0 \\
\hline Psychiatry & 6479 & 5.2 & 3477 & 10.2 \\
\hline Pulmonary & 13 & 0.0 & 14 & 14.3 \\
\hline Rheumatology & 69 & 14.5 & 712 & 11.8 \\
\hline Urology & 224 & 5.8 & 1631 & 9.1 \\
\hline
\end{tabular}

Table 3 Rates of genetic testing by FDA-define therapeutic area. for those who did not $(\$ 1331 ; p<0.0001)$. Similarly, all other cost comparisons were significant at the same level, with the PMPM costs for the proactive genetic testing group (\$1582; 95\% CI: \$1432-1747) exceeding those of their matched comparators (\$1289; 95\% CI: \$1166-1425) and the same being true for the reactive testing group ( $\$ 1746$ [95\% CI: \$1509-2019] vs. \$1405 [95\% CI: \$1218-1620]). Unlike the Medicaid cohort, however, comparisons between the proactive and reactive groups indicated significantly higher monthly expenditures for individuals receiving reactive genetic testing $(p<0.0001)$. More detailed information about cohort-specific PMPM costs is contained in Table 5 and a list of all target medications/frequencies appears in Appendix 3.

Data regarding the rates of genetic testing by therapeutic area in the Medicare cohort can also be seen in Table 3 . Consistent with the overall results, category-specific genetic testing occurred approximately twice as often in the Medicare cohort in comparison to the Medicaid cohort for most therapeutic areas. Oncology drugs were an exception to this general rule with $40.0 \%$ of patients receiving genetic 
testing, as were rheumatology drugs with more frequent testing in the Medicaid cohort (although from a low number of prescriptions; $n=69$ ). The level of disparity was also much wider across the two cohorts for gastroenterology

Table 4 Medicare cohort demographics.

\begin{tabular}{|c|c|c|c|}
\hline \multicolumn{4}{|l|}{ Total cohort } \\
\hline & Genetic testing & $\begin{array}{l}\text { No genetic } \\
\text { testing }\end{array}$ & $p$-value \\
\hline & $n=3960$ & $n=33,828$ & \\
\hline Race, $n(\%)$ & & & 0.1396 \\
\hline Black & $870(22.0)$ & $7906(23.4)$ & \\
\hline White & $3038(76.7)$ & $25,497(75.4)$ & \\
\hline Other & $52(1.3)$ & $425(1.3)$ & \\
\hline Gender, $n(\%)$ & & & $<0.0001$ \\
\hline Male & $1085(27.4)$ & $11,293(33.4)$ & \\
\hline Female & $2875(72.6)$ & $22,535(66.6)$ & \\
\hline Low-income subsidy & & & 0.8894 \\
\hline Yes & $1451(36.6)$ & $12,357(36.5)$ & \\
\hline No & $2509(63.4)$ & $21,471(63.5)$ & \\
\hline Age, mean (SD) & $75.0(6.8)$ & $75.9(7.4)$ & $<0.0001$ \\
\hline $\begin{array}{l}\text { Elixhauser score, } \\
\text { mean (SD) }\end{array}$ & $4.19(2.8)$ & $3.23(2.6)$ & $<0.0001$ \\
\hline \multicolumn{4}{|c|}{ Final cohort with matched comparators } \\
\hline & Genetic testing & $\begin{array}{l}\text { No genetic } \\
\text { testing }\end{array}$ & $p$-value \\
\hline & $n=3851$ & $n=3851$ & \\
\hline Race, $n(\%)$ & & & 0.0129 \\
\hline Black & $840(21.8)$ & $952(24.7)$ & \\
\hline White & $2959(76.8)$ & $2852(74.1)$ & \\
\hline Other & $52(1.4)$ & $47(1.2)$ & \\
\hline Gender, $n(\%)$ & & & $<0.0001$ \\
\hline Male & $1055(27.4)$ & $1225(31.8)$ & \\
\hline Female & $2796(72.6)$ & $2626(68.2)$ & \\
\hline Low-income subsidy & & & 0.0477 \\
\hline Yes & 1409 (36.59) & 1492 (38.74) & \\
\hline No & $2442(63.41)$ & $2359(61.26)$ & \\
\hline Age, mean (SD) & $75.0(6.8)$ & $76.3(7.5)$ & $<0.001$ \\
\hline $\begin{array}{l}\text { Elixhauser score, } \\
\text { mean (SD) }\end{array}$ & $4.11(2.7)$ & $4.11(2.7)$ & - \\
\hline
\end{tabular}

drugs, with $10.6 \%$ of the Medicare cohort receiving genetic testing for these commonly prescribed medications (compared to $2.9 \%$ in the Medicaid cohort).

\section{Discussion}

The first broad conclusion of the study is that few people received genetic testing, despite a nearly 2.5 -times difference between the Medicaid (4.4\%) and Medicare (10.5\%) cohorts. This is potentially meaningful in terms of estimating the degree of permeation of contemporary pharmacogenomic science into clinical practice, in that $100 \%$ of the individuals selected were taking a medication for which information about genetic testing appeared in the drug's FDA-approved labeling. The combination of this inclusion criterion with the broad set of CPT codes used to depict genetic testing of any kind cast the widest possible net to determine what was essentially a ceiling effect of the likelihood of receiving genetic testing. Unfortunately, it appeared that this ceiling was very low and that there were barriers in translating FDA labeling information into behavioral change for providers and/or consumers of medical services when and where this was warranted (consistent with the low testing rates seen in previous examinations) [1-4].

In addition, these results were obtained from the entire population of enrollees in Medicare and Medicaid, which together comprised over 39\% of all Mississippi residents in 2014 [22-24]. On the basis of this extremely representative group it was apparent that there were large demographic differences in testing in both cohorts, with White and female beneficiaries exhibiting a much higher frequency. In the case of differences between ethnic groups, this is consistent with both a lack of ethnic diversity in pharmacogenomic research $[25,26]$ and a wider medical literature reflecting similar disparities and barriers to service accessibility in minority groups (most recently exemplified and made even more salient in the COVID-19 pandemic) [27-31]. It was less clear why this would be the case for gender, particularly considering that a perusal of the target drugs in Appendixes 2 and 3 did not reveal a differential frequency of medications designed to treat diseases exclusively or primarily seen in females (e.g., breast or uterine cancer).
Table 5 Mississippi Medicare adjusted all-cause per member per month (PMPM) costs.

\begin{tabular}{lllllll}
\hline Cohort & Genetic testing & & & No genetic testing & \multirow{2}{*}{$p$-value } \\
\cline { 2 - 3 } & PMPM cost & $95 \%$ CI & & PMPM cost & $95 \%$ CI & \\
\hline Total sample & $1637(n=3851)$ & $1507-1778$ & & $1331(n=3851)$ & $1226-1445$ & $<0.0001$ \\
Proactive testing & $1582(n=2814)$ & $1432-1747$ & & $1289(n=2814)$ & $1166-1425$ & $<0.0001$ \\
Reactive testing & $1746(n=1037)$ & $1509-2019$ & & $1405(n=1037)$ & $1218-1620$ & $<0.0001$ \\
\hline
\end{tabular}

The difference between proactive and reactive testing subgroups was significant; $p<0.0001$. 
The differences in adjusted all-cause PMPM costs included significantly higher costs for the testing group vs. matched comparators across both cohorts, as well as differences within-cohort comparing those receiving proactive vs. reactive testing. In the Medicaid cohort, this difference indicated higher expenditures for the proactive testing group, whereas the opposite was seen in the Medicare cohort. The reasons for these collective findings cannot be directly informed by the current study, although it is possible that differences in expenditures reflected clinical decisions related to symptom severity (which could not be captured in the current study). Serious presentations of a given condition could have been referred for genetic testing more frequently due to the perception of greater risk inherent in certain treatment approaches (and thus the need for more caution/attention to labeling warnings in ascertaining likely ADRs in advance). Although not directly informed by the current study due to the use of claims data, the idea that clinicians are recommending testing differentially on the basis of some patient characteristics (e.g., the severity of presenting conditions) would suggest that providers were at least aware/becoming aware of the possible clinical advantages of testing. This is encouraging, given the notable time lag between the development and implementation of pharmacogenomic technologies and the numerous identified barriers to dissemination [32-37]. Time and further research will be necessary to elucidate a trajectory for how quickly this awareness will spread and translate into behavioral change, but knowledge of innovation is a necessary first step in eventual adoption for habitual use [38]. Conducting studies of this nature is challenging, however, given the diverse, complex, and sometimes contradictory information available from relevant scientific summaries, FDA labeling information, and professional organizational policy statements regarding genetic testing. Thus, tracing the impact of research findings on individual clinical decisions is likely to be an intricate, difficult process that will require much more focused, prospective data collection to examine with any degree of specificity.

Finally, comparisons across drugs from different therapeutic areas indicated general consistency of within-cohort base rates of genetic testing with the notable exception of oncology medications. Although these comprised a relatively small percentage of overall medications prescribed in each cohort, the rate of testing was substantially higher for these drugs than any other (36.4\% in Medicaid and $40.0 \%$ in Medicare). Although it is difficult to infer generalizable conclusions from the limited subset of prescriptions and the highly selected nature of participants, this finding is also encouraging in terms of pharmacogenomic research influencing applied practice. Testing may have been mandated in many cases (given the nature of modern cancer treatment agents and policies regarding their prescription) [39], but regardless, the greatly increased frequency of testing for these drugs suggested some differential influence on oncology clinicians' decision-making processes.

\section{Limitations}

The study is not without limitations, particularly in terms of the low frequency of genetic testing. Any findings of the group of people receiving these tests are potentially difficult to extrapolate given that they are based on a small percentage of those taking the target medications. This is further compounded by the diversity of health conditions treated by drugs with related FDA labeling information, which potentially led to a non-homogenous sample in terms of initial symptoms (although the within-category findings were fairly similar for most drugs other than oncology). Given a large enough base rate of any single condition or medication, it might be possible to extend this work to a relatively uniform sample with similar presenting concerns, but the current data set did not afford this possibility. Further, even when a CPT for genetic testing was present in a beneficiary's record it was not possible to know if it occurred in relation to the target medication or for other purposes. In addition, the follow-up period for both cohorts was fairly short, which did not allow a more nuanced appraisal of potential economic benefits associated with testing. If pharmacogenomic testing is functioning within this system in the way it is meant to function (i.e., to avoid ADRs and enable individually tailored precision interventions) then costs savings may not be realized until farther away from the medical issue or episode that provided the impetus for testing.

\section{Future directions}

Future studies of more direct inquiry into physician and patient decision-making about the need for pharmacogenomic testing may elucidate the rationale for such low frequencies among groups with direct, salient indicators that testing could contribute positively to clinical prediction. This might entail the review of medical records, interviews with physicians and patients, assessment of follow-through on medical advice, and/or studies to understand more about how people react to pharmacogenomic feedback. This latter point may be particularly fruitful if test manufacturers design decision support tools [40-45] to convey results in terms that both providers and patients can quickly understand, as the lack of comprehensibility could be a barrier to further dissemination.

In addition, studies focused on costs (particularly longterm costs) and health outcomes associated with genetic testing may be particularly useful in convincing large 
healthcare organizations to develop policies and specific mandates for their use. Taking a system focus for these future inquiries it may also be possible to develop simple, automated, administrative feedback tools to encourage implementation of testing and aggregated use of results. Knowing who should be tested, why, how to make clinical use of the results, and the relative costs (or savings) to the system in terms of all decision points may allow a more specific, pragmatic interpretation of the value of pharmacogenomic testing in practice. In so doing, cost differentials might be clarified and ADRs, rehospitalizations, deaths, and/or other non-optimal outcomes may be reduced.

\section{Conclusion}

This study demonstrated that pharmacogenomic testing is relatively uncommon in public payer healthcare systems, even in the context of beneficiaries taking medications with labeling information denoting the potential benefits of such testing. Despite the emergence of some predictive variables in terms of enhancing the likelihood of undergoing testing, the base rate of usage and pattern of results was not sufficient to allow attribution of differences to specific causes. Additional research focused on more direct measurement of testing practice, patient follow-through, and interpretation/use of results may provide greater insights about the nature of barriers to implementing genetic testing in applied practice.

\section{Compliance with ethical standards}

Conflict of interest The authors declare that they have no conflict of interest.

Publisher's note Springer Nature remains neutral with regard to jurisdictional claims in published maps and institutional affiliations.

\section{References}

1. Phillips KA. Evolving payer coverage policies on genomic sequencing tests: beginning of the end or end of the beginning? JAMA 2018;319(Jun):2379-80.

2. Phillips KA, Deverka PA, Hooker GW, Douglas MP. Genetic test availability and spending: where are we now? Where are we going? Health Aff. 2018;37:710-6.

3. Lynch JA, Berse B, Dotson DW, Khoury MJ, Coomer N, Kautter J. Utilization of genetic tests: analysis of gene-specific billing in Medicare claims data. Genet Med. 2017;19:890.

4. De Sa J, Carlson B, Caputo N, Vojta D, Sandy L, Stevens S. Growth of molecular diagnostics and genetic testing in the USA, 2008-2011: analysis and implications. Pers Med. 2013;10 (Nov):785-92.

5. Alagoz O, Durham D, Kasirajan K. Cost-effectiveness of one-time genetic testing to minimize lifetime adverse drug reactions. Pharmacogenom J. 2015;16:129-36.
6. Phillips KA, Veenstra DL, Oren E, Lee JK, Sadee W. Potential role of pharmacogenomics in reducing adverse drug reactions: a systematic review. JAMA 2001;286(Nov):2270-9.

7. Hallberg P, Yue QY, Eliasson E, Melhus H, Ås J, Wadelius M. SWEDEGENE - a Swedish nation-wide DNA sample collection for pharmacogenomic studies of serious adverse drug reactions. Pharmacogenom J. 2020;20:579-85.

8. von Euler M, Eliasson E, Öhlén G, Bergman U. Adverse drug reactions causing hospitalization can be monitored from computerized medical records and thereby indicate the quality of drug utilization. Pharmacoepidemiol Drug Saf. 2006;15(Mar):179-84.

9. Lundkvist J, Jönsson B. Pharmacoeconomics of adverse drug reactions. Fund Clin Pharm. 2004;18(Jun):275-80.

10. Wester K, Jönsson AK, Spigset O, Druid H, Hägg S. Incidence of fatal adverse drug reactions: a population based study. Br J Clin Pharm. 2008;65(Apr):573-9.

11. Planelles B, Margarit C, Inda MM, Ballester P, Muriel J, Barrachina $\mathbf{J}$, et al. Gender based differences, pharmacogenetics and adverse events in chronic pain management. Pharmacogenom J. 2020;20:320-8.

12. Severino G, Del Zompo M. Adverse drug reactions: role of pharmacogenomics. Pharm Res. 2004;49(Apr):363-73.

13. Zhou X, Hetrick SE, Cuijpers P, Qin B, Barth J, Whittington CJ, et al. Comparative efficacy and acceptability of psychotherapies for depression in children and adolescents: a systematic review and network meta-analysis. World Psychiatry. 2015;14:207-22.

14. Chan SL, Suo C, Lee SC, Goh BC, Chia KS, Teo YY. Translational aspects of genetic factors in the prediction of drug response variability: a case study of warfarin pharmacogenomics in a multiethnic cohort from Asia. Pharmacogenom J. 2012;12(Aug):312-8.

15. Brixner D, Biltaji E, Bress A, Unni S, Ye X, Mamiya T, et al. The effect of pharmacogenetic profiling with a clinical decision support tool on healthcare resource utilization and estimated costs in the elderly exposed to polypharmacy. J Med Econ. 2015;19:213-28.

16. Bachtiar M, Ooi BN, Wang J, Jin Y, Tan TW, Chong SS, et al. Towards precision medicine: Interrogating the human genome to identify drug pathways associated with potentially functional, population-differentiated polymorphisms. Pharmacogenom J. 2019;19(Dec):516-27.

17. US Food and Drug Administration. Table of pharmacogenomic biomarkers in drug labeling. https://www.fda.gov/drugs/science-a nd-research-drugs/table-pharmacogenomic-biomarkers-drug-la beling. Accessed $20 \mathrm{Jul} 2020$.

18. Ohara K. CPT code updates for 2012. Clinical terminologies \& coding and reimbursement. http://library.ahima.org/doc?oid= 105353\#.XxX6cy05RUM (2012). Accessed 20 Jul 2020.

19. Hefti E, Blanco J. Documenting pharmacogenomic testing with current procedure terminology (CPT) codes, a review of past and present practices. Health Inf Manag J. 2016;87:56-9.

20. Young J, Ramachandran S, Freeman AJ, Bentley JP, Banahan BF. Patterns of treatment for psychiatric disorders among children and adolescents in Mississippi Medicaid. PLoS ONE. 2019;14: $\mathrm{e} 0221251$.

21. van Walraven C, Austin PC, Jennings A, Quan H, Forster AJ. A modification of the Elixhauser comorbidity measures into a point system for hospital death using administrative data. Med Care. 2009;47:626-33.

22. KFF. Medicaid enrollees by enrollment group. https://www.kff. org/medicaid/state-indicator/distribution-of-medicaid-enrolleesby-enrollment-group/?currentTimeframe $=0 \&$ sortModel $=\% 7 \mathrm{~B} \%$ 22colId\%22:\%22Location \%22,\%22sort\%22:\%22asc\%22\%7D (2020). Accessed $20 \mathrm{Jul} 2020$.

23. CMS. Original Medicare enrollment. https://www.cms.gov/Resea rch-Statistics-Data-and-Systems/Statistics-Trends-and-Reports/ CMSProgramStatistics/2014/Downloads/MDCR_ENROLL_AB/ 
2014_CPS_MDCR_ENROLL_AB_13.pdf (2014). Accessed 20 Jul 2020.

24. United States Census Bureau. QuickFacts Mississippi. https://www.census.gov/quickfacts/fact/table/MS/PST120219 (2019). Accessed 20 Jul 2020.

25. De T, Park CS, Perera MA. Cardiovascular pharmacogenomics: does it matter if you're Black or White? Ann Rev Pharm. 2019;59 (Jan):577-603.

26. Popejoy AB, Fullerton SM. Genomics is failing on diversity. Nature. 2016;538(Oct):161.

27. Wadhera RK, Wadhera P, Gaba P, Figueroa JF, Maddox KE, Yeh RW, et al. Variation in COVID-19 hospitalizations and deaths across New York city boroughs. JAMA. 2020;323:2192-915.

28. Maina IW, Belton TD, Ginzberg S, Singh A, Johnson TJ. A decade of studying implicit racial/ethnic bias in healthcare providers using the implicit association test. Soc Sci Med. 2018;199:219-29.

29. Hall WJ, Chapman MV, Lee KM, Merino YM, Thomas TW, Payne BK, et al. Implicit racial/ethnic bias among health care professionals and its influence on health care outcomes: a systematic review. Am J Pub Health. 2015;105(Dec):e60-76.

30. Yancy CW. COVID-19 and African Americans. JAMA. 2020;323:1891-2.

31. Williams DR, Cooper LA. COVID-19 and health equity-a new kind of herd immunity. JAMA 2020;323:2478-80.

32. Amara N, Blouin-Bougie J, Bouthillier D, Simard J. On the readiness of physicians for pharmacogenomics testing: an empirical assessment. Pharmacogenom J. 2018;18:308-18.

33. Dias MM, Ward HM, Sorich MJ, McKinnon RA. Exploration of the perceptions, barriers and drivers of pharmacogenomics practice among hospital pharmacists in Adelaide, South Australia. Pharmacogenom J. 2014;14:235-40.

34. Hoffman JM, Flynn AJ, Juskewitch JE, Freimuth RR. Biomedical data science and informatics challenges to implementing pharmacogenomics with electronic health records. Ann Rev Biomed Data Sci. 2020;3.
35. Klein ME, Parvez MM, Shin J-G. Clinical implementation of pharmacogenomics for personalized precision medicine: barriers and solutions. J Pharm Sci. 2017;106:2368-79.

36. Mikat-Stevens NA, Larson IA, Tarini BA. Primary-care providers' perceived barriers to integration of genetics services: a systematic review of the literature. Genet Med. 2014;17:169-76.

37. Waldman L, Shuman C, Cohn I, Kaiser A, Chitayat D, Wasim S, et al. Perplexed by PGx? Exploring the impact of pharmacogenomic results on medical management, disclosures and patient behavior. Pharmacogenomics 2019;20:319-29.

38. Rogers E. Diffusion of innovations. New York: Free Press; 2003.

39. Robson ME, Bradbury AR, Arun B, Domchek SM, Ford JM, Hampel HL, et al. American society of clinical oncology policy statement update: genetic and genomic testing for cancer susceptibility. J Clin Oncol 2015;33:3660-67.

40. Abbott R, Chang DD, Eyre HA, Bousman CA, Merrill D, Lavretsky $\mathrm{H}$. Pharmacogenetic decision support tools: a new paradigm for late-life depression? Am J Geriatr Psychiatry. 2018;26:125-33.

41. Bousman CA, Dunlop BW. Genotype, phenotype, and medication recommendation agreement among commercial pharmacogeneticbased decision support tools. Pharmacogenom J. 2018;18:613-22.

42. Bousman CA, Hopwood M. Commercial pharmacogenetic-based decision-support tools in psychiatry. Lancet Psychiatry. 2016;3:585-90.

43. Hall-Flavin DK, Winner JG, Allen JD, Carhart JM, Proctor B, Snyder KA, et al. Utility of integrated pharmacogenomic testing to support the treatment of major depressive disorder in a psychiatric outpatient setting. Pharmacogenet Genom. 2013;23:535-48.

44. Hicks JK, Dunnenberger HM, Gumpper KF, Haidar CE, Hoffman JM. Integrating pharmacogenomics into electronic health records with clinical decision support. Am J Health 2016;73:1967-76.

45. Hicks JK, Stowe D, Willner MA, Wai M, Daly T, Gordon SM, et al. Implementation of clinical pharmacogenomics within a large health system: from electronic health record decision support to consultation services. Pharmacother J Hum Pharmacol Drug Ther. 2016;36:940-8. 